\title{
Knowledge Granularity and Representation of Knowledge: Towards Knowledge Grid
}

\author{
Maria A. Mach and Mieczyslaw L. Owoc \\ Wroclaw University of Economics \\ Komandorska 118/120 \\ 53-345 Wroclaw, Poland \\ \{maria.mach, mieczyslaw.owoc\} @ue.wroc.pl
}

\begin{abstract}
Knowledge granularity, usually identified with the size of knowledge granules, seems to be real challenge for knowledge consumers as well as for knowledge creators. In this paper, relationships between knowledge granularity as a result of different ways of a knowledge representation are considered. The paper deals with the problem of developing knowledge grid in the context of encapsulation of knowledge including different dimensions and measures. The origin of the problem is discussed in the first section stressing flexibility of knowledge interpretations. Concepts of knowledge granularity (also from formal point of view) are presented in the next section. The nature of well represented knowledge is considered in the next chapter with references to granularity of knowledge. In the last part of the paper the question of formulating knowledge granularity in the context of knowledge grid concepts is discussed. This document comprising guidelines for authors is divided into several sections.
\end{abstract}

Keywords: Knowledge grid, representation of knowledge, knowledge granularity.

\section{Introduction}

Knowledge as the essential term defined in many ways (stressing its philosophical or domain roots and aspects) represents structured information with the ability of interpretation and application for different purposes. The most general knowledge definition states, that knowledge may be perceived as: (i) expertise, and skills acquired by a person through experience or education; the theoretical or practical understanding of a subject; (ii) what is known in a particular field or in total; facts and information; or (iii) awareness or familiarity gained by experience of a fact or situation. In the field of artificial intelligence, the second meaning of the notion is common. Nevertheless of the context discussed, knowledge should be considered as the crucial value creating rational base for the future acting for potential users (individual or organizational). This is worth to stress that in different areas of knowledge applications we mainly use some pieces of knowledge for performing particular tasks. On the other hand there is a natural tendency to define whole knowledge which is useful in supporting selected 
activities in the broadly understood decision-making processes. Therefore a quest of knowledge scopes essential and useful in such a context seems to be obvious.

The main goal of the paper is to investigate solutions in defining knowledge pieces (as a part of the whole relevant knowledge) in order to support knowledge grid concepts. Considering explicit knowledge, which is available for different users, we tend to focus on elaborated knowledge representation techniques acceptable in the computer infrastructure. Knowledge grid identified with intelligent and available via computer network platform covering explicit knowledge resources and supporting ondemand services oriented on problem solving, decision-making and the like processes (comp. [Zhuge, 2002]). Therefore knowledge granularity, knowledge representation and knowledge grid are the crucial categories in this research.

\section{Concepts of Knowledge Granularity}

The concept of granularity (in general) comes from photography, and describes accuracy of pictorial presentation on film. In a more specific way, one may speak also of information resources granularity and knowledge granularity.

Granularity of information resources refers to size, decomposability, and extent to which a resource is intended to be used as a part of a larger one. A typical example of information resources granularity concerns hierarchy of data, as depicted in Fig. 1.

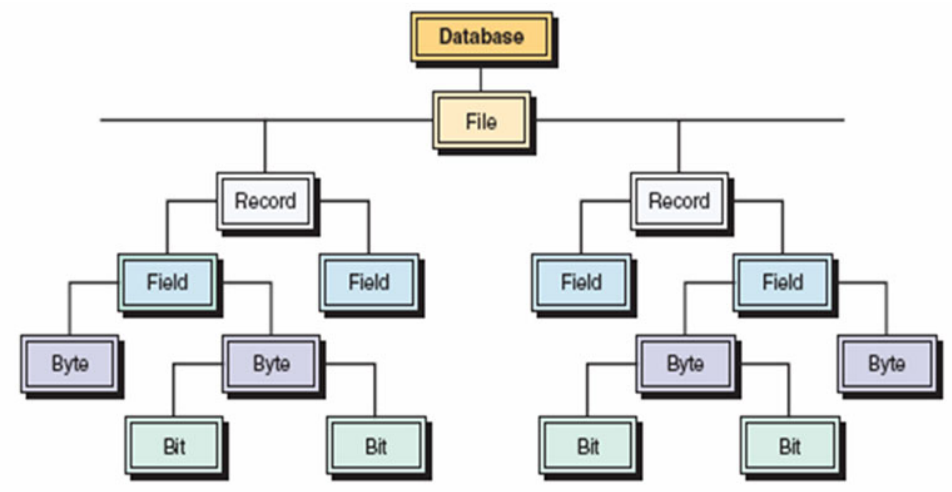

Fig. 1. Hierarchy of data as granularity of data

Source: [Turban et al., 2008], p. 88.

Knowledge granularity, in turn, is linked with dividing knowledge into pieces (socalled knowledge chunks), i.e. perceiving different levels of detail in knowledge structures.

The origins of knowledge granularity phenomenon are threefold. First, knowledge in modern information systems comes from many sources and is mapped in many ways. Second, there are many different forms of presentation of knowledge (e.g. semantic networks, rules, frames etc.). Finally, there are different domains of knowledge application: for example, different knowledge is needed on different management 
levels - a CEO needs more "concentrated" knowledge, while a bottom-level user needs more details.

To the best of our knowledge, there is no formal specification of knowledge granularity in the literature. For the purposes of this paper, we adopted Bettini's formalization of time granularity (may be found in [Bettini et al., 1998]). Not all the concepts of time granularity apply to knowledge granularity, because knowledge is of different nature than time. Nevertheless, some of Bettini's concepts may be used.

By knowledge domain we understand a set of primitive knowledge entities concerning a particular problem. These entities may be for example raw data. All coarser knowledge chunks are generated from these primitive entities.

Knowledge granularity may be formally defined as the resolution power of qualification of a knowledge piece. A granularity is a mapping $\mathrm{G}$ from integers to subsets of knowledge domain, such that (see also [Bettini et al., 1998b]):

1. If $\mathrm{i}<\mathrm{j}$ and $\mathrm{G}(\mathrm{i}), \mathrm{G}(\mathrm{j})$ are non-empty, then each element of $\mathrm{G}(\mathrm{i})$ is less than all elements of $G(j)$. This is to state that granularities do not overlap;

2. If $\mathrm{i}<\mathrm{k}<\mathrm{j}$ and $\mathrm{G}(\mathrm{i}), \mathrm{G}(\mathrm{j})$ are non-empty, then $\mathrm{G}(\mathrm{k})$ is non-empty. This rule means that the subset of the index set that maps to non-empty subsets of knowledge domain is contiguous. Each non-empty subset G(i) is called a granule of granularity G. A granule may be composed e.g. of data.

Granularities and granules are interrelated in many ways. We may adopt three kinds of relationships from Bettini's framework:

1. Groups into - a granularity $\mathrm{G}$ groups into a granularity $\mathrm{H}(\mathrm{G} \backslash \mathrm{H})$ if for each index $j$ there exists a subset $S$ of the integers such that $H(j)=\cup_{i \in S} G(i)$.

Example: granules of type "data" and "data label" group into a granule of type "information" (where information $=$ data + label $)$

2. Finer than - a granularity $\mathrm{G}$ is finer than a granularity $\mathrm{H}(\mathrm{G} \leq \mathrm{H})$ if for each index $i$, there exists an index $j$ such that $\mathrm{G}(\mathrm{i}) \subseteq \mathrm{H}(\mathrm{j})$.

Example: information on levels of sugar measured in blood (daily measure) is finer than information on HbA1c (level of sugar in a three-month period); a list of individual salaries is finer than average salaries grouped by department.

3. Partitions - a granularity $\mathrm{G}$ partitions a granularity $\mathrm{H}$, if $\mathrm{G} \varangle \mathrm{H}$ and $\mathrm{G} \leq \mathrm{H}$.

Having granularities and granules of knowledge, one may perform some kind of granular reasoning. This concerns using some particular functions that operate on granules. These functions enable:

- creating queries concerning main granular components (e.g. granular levels),

- extracting contents of levels,

- moving across levels, e.g. if we compute average salary of each department, we move from a more detailed (i.e. finer) knowledge level onto a coarser one.

We are aware that many knowledge representation methods are based on hierarchical classification, for example description logic based knowledge representation. Nevertheless using knowledge granularity concepts one may not only move across levels 
and use different "pieces" of knowledge, but may also enrich the representation with the notion of time, a very important aspect of changing world.

Presented in brief the concept of knowledge granularity should convince us about great importance of implementing "knowledge pieces" in reality.

\section{Selecting Knowledge Granularity in the Knowledge Representation Context}

There is a natural tend to represent any part of reality (perhaps anything) sometimes called as upper ontology where generalized view of the world consists of intuitively formulated objects. Figure 2 depicts one of possible hierarchies representing of anything.

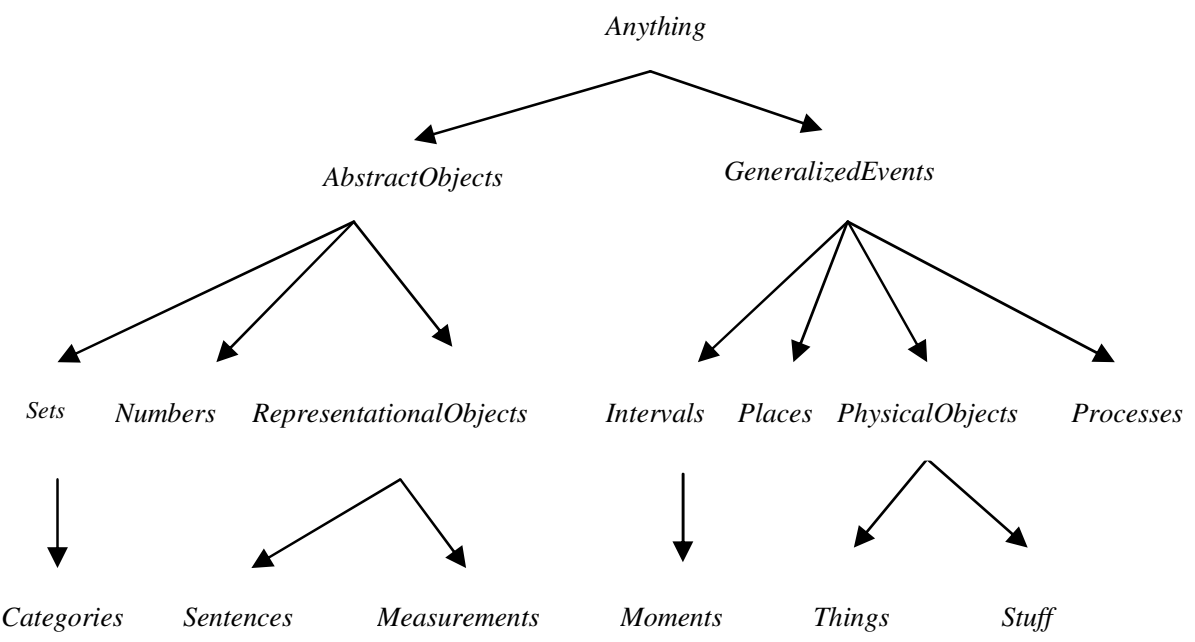

Fig. 2. The upper ontology of the world

Source: [Russell and Norvig, 2003], p.321

Concepts introduced in Figure 2 express in a simplified way a hierarchy of components existing in any domain covering static (AbstractObjects) as well as dynamic aspects of its functioning (GeneralizedEvents). At the upper level of ontology discussed we focus on unification of represented knowledge which allows for applying reasoning and problem solving techniques in more universal way.

Let us remind the main assumptions of properly represented knowledge. Nevertheless the techniques of knowledge representation we need to organize objects or events into categories which are interrelated in some way. Both sides of the hierarchy expressed in Figure covering selected details referring to AbstractObjects and GeneralizedEvents. This way materialized knowledge can be used through inheritance of features from higher levels of abstraction (e.g. Sentences and Measurements in case of 
RepresentationalObjects or Things and Staff for PhysicalObjects). At any level of knowledge representation process we have to decide about knowledge granularity.

Knowledge granularity concept defined earlier can be used in practice for example in search agent purpose (see: Yiming and Tsotsos, 2001). Agents performing determined tasks use certain kind of knowledge recall single granularity or a set of hierarchical granularities in various situations. In some circumstances an agent is able to select the best knowledge granularity according to maximizing its performance. In the complex agent environment particular agent is able to apply granularity representing the corresponding knowledge from the demanding environment-granularity hash table.

The presented example of the "working" knowledge granularity in agent systems confirms usability of this approach. Keeping in memory some features of knowledge granularity we are able to select proper representation schema for the defined purpose.

\section{Knowledge Grid and Knowledge Granularity}

The concept of knowledge granularity can be applied in these approaches where we need to represent knowledge pieces in more flexible way. Therefore natural references of knowledge granularity can be found in the knowledge grid context.

According to quoted earlier definition proposed by $\mathrm{H}$. Zhuge we assume that knowledge grid "is an intelligent and sustainable Internet application environment that enables people or virtual roles to effectively capture, coordinate, publish, understand, share and manage knowledge resources" (see: [Zhuge 2004]). Ultimate goal of knowledge grid systems is to provide services to support innovation, problem solving and decision making in a distributed environment, which varies in scale and stability.

For this purpose the need of developing a new architecture seems to be obvious. The Knowledge Grid as a new architecture based on the existing methods and technologies such as the Grid, the Semantic Web, Web Services Peer-to-Peer, AI, proper data modelling, information processing technologies and system methodology. Such system can be formulated as a three-layer model (see: [Hengsham, Liqun 2005]) consisting of the following layers:

- $\quad$ knowledge storage layer,

- $\quad$ knowledge services layer,

- $\quad$ knowledge grid application with interface for the end-user.

The knowledge storage layer corresponds mostly to the particular knowledge domain database. The pieces of knowledge are stored and can be acquired from there. This layer's function is to provide secure access to the knowledge items. Therefore all the mentioned before relationships in terms of knowledge granularity are actual: groups into, finer than and partitions. We create specific repository of knowledge assuming expecting level of knowledge granularity. Of course all necessary functions assuring knowledge storage functionality should be involved: syntactic analysis, searching, inquiring and extending knowledge bases.

The knowledge services layer supplies one view of heterogeneous knowledge sources and software systems, together with suitable software for knowledge discovery and reduction of redundant information. Furthermore, knowledge in this layer is used to improve query precision and to explain results to the end-user. The processes, 
which help to intelligently eliminate, create and discover organisational knowledge, happen in this middle layer. All potential services are direct oriented on knowledge granules in terms of performing the itemized processes.

The knowledge grid application layer is responsible for delivering knowledge to a particular user. As before processes formulated by users recall some pieces of knowledge from the storage level. Again relationships among the formulated earlier knowledge granules determine ways of performing tasks by a user.

The presented in the paper concepts of knowledge granularity and knowledge grid create intersection of the research streams and confirm intuitively formulated relationships. Let us stress the placement of both concepts in broadly understood research areas of knowledge grid (compare: Zhuge 2004 and Owoc 2009):

- Theories and methods for supporting knowledge management. Defining the core phases of knowledge management: gathering, representation and sharing knowledge we should express the expecting level of knowledge granularity in the knowledge management process. The value of the knowledge grid approach in knowledge management solutions is common accepted from many reasons.

- Ontology and semantic aspects of knowledge grid. Nevertheless of the knowledge grid application area there are huge of problems with common understanding of the whole concept; domain terminology, interpretation of interrelationships, principles and references to other disciplines including flexible understanding of knowledge granularity concepts.

- Knowledge grid in different institutions. Propagation and management of knowledge within a virtual organization is one of the suggested hot topics. There are problems how implement knowledge grid in global organizations and on the other hand what kind of information infrastructure could be effective in case of hybrid and multilevel companies. One can assume specific approaches to formulating knowledge granularity in different companies.

- knowledge grid and effectiveness of knowledge management. Taking into consideration particular knowledge management phases: organization, evaluation, and improvement we are looking for efficient tools and techniques in order to support the whole cycle. The knowledge grid seems to be very promising in eliminating redundant knowledge and improving knowledge so that quality of useful knowledge pieces and knowledge as a whole should be better and better. It covers also such processes like: creation new knowledge from existing well-represented knowledge, from case histories, and from raw knowledge materials like texts. The role of knowledge granularity in these processes is very important.

Knowledge integration in the grid architecture. One of the promising features of the presented approach is gathering information and knowledge pieces from many sources. For example integrating knowledge resources could support analogies, problem solving, and scientific discovery so standards in this area are welcome. In every case we should define a proper lever of knowledge granularity. 


\section{Conclusions}

This paper presents intuitively observed correlation between knowledge granularity and knowledge grid. Main research findings can be formulated in the following way:

1. Adopted from Bettini's framework, concept of granularity can be applied to formulating of knowledge granularity concepts. This approach can be compared with other proposals of knowledge granularity. Especially relationships formulated in this framework are useful in different domains.

2. Knowledge granularity is the very important component of broadly understood knowledge representation process. In more universal concept of the upper ontology of the world knowledge granularity plays the essential role.

3. In knowledge grid approaches a concept of knowledge granularity is present in many research areas. It is very difficult to create and manage knowledge grid without properly defined knowledge granularity concepts.

The further research can embrace investigation on determining standards and dimensions of knowledge granularity in knowledge grid architectures applied in different areas.

\section{References}

Bettini, C., Wang, X., Jajodia, S.: A General Framework for Time Granularity and its Application to Temporal Reasoning. Annals of Mathematics and Artificial Intelligence 22(1,2), 29 58 (1998)

Bettini, C., Dyreson, C.E., Evans, W.S., Snodgrass, R.T., Wang, X.S.: A Glossary of Time Granularity Concepts. In: Etzion, O., Jajodia, S., Sripada, S. (eds.) Dagstuhl Seminar 1997. LNCS, vol. 1399, pp. 406-413. Springer, Heidelberg (1998)

Bittner, T., Smith, B.: A Theory of Granular Partitions. In: Foundations of Geographic Information Science

Duckham, M., Goodchild, M.F., Worboys, M.F. (eds.): Taylor \& Francis Books, London (2003)

Benett, B.: Space, Time, Matter and Things. In: Proc. FOIS 2001. ACM Press, USA (2001)

Bettini, C., Jajodia, S., Wang, S.X.: Time Granularities in Databases. In: Data Mining, and Temporal Reasoning, Springer, Berlin (2000)

Duncan, C.: Granularisation. In: Littlejohn, A. (ed.) Reusing Online Resources: A Sustainable Approach to eLearning. Kogan Page, London (2003)

Feng, Q., Miao, D., Zhou, J., Cheng, Y.: A Novel Measure of Knowledge Granularity in Rough Sets

Goralwalla, I.A., Leontiev, Y., Ozsu, T.M., Szafron, D.: Temporal Granularity: Completing the Puzzle. Journal of Intelligent Information Systems 16, 41-46 (2001)

Huang, F., Zhang, S.: Clustering Web Documents Based on Knowledge Granularity. Asian Journal on Information Technology 5(1) (2006) 
Keet, C.M.: A Formal Theory of Granularity. PhD Thesis, KRDB Research Centre, Faculty of Computer Science, Free University of Bozen-Bolzano, Italy (2008)

Keet, C.M.: A taxonomy of types of granularity. In: IEEE Conference in Granular Computing (GrC 2006), Atlanta, USA, May 10-12 (2006)

Kamble, A.S.: A Data Warehouse Conceptual Data Model for Multidimensional Information. $\mathrm{PhD}$ thesis, University of Manchester, UK (2004)

Mach, M.A., Owoc, M.L.: Granularity of Knowledge from Different Sources. In: Intelligent Information Processing IV, IFIP - The International Federation for Information Processing, vol. 288 (2009)

Mach, M., Owoc, M.L.: About Dimension and Measures of Knowledge Granularity. In: Tadeusiewicz, R., Ligeza, A., Szymkat, M. (eds.) Computer Methods and Systems, Kraków, vol. I (2009)

Mani, I.: A theory of granularity and its application to problems of polysemy and underspecification of meaning. In: Cohn, A.G., Schubert, L.K., Shapiro, S.C. (eds.) Proceedings of the Sixth International Conference on Principles of Knowledge Representation and Reasoning, KR 1998. Morgan Kaufmann, San Mateo (1998)

Owoc, M.L.: Research trends in knowledge grid. In: Nowicki, A. (ed.) Business Informatics 13. Research Papers No 55 of Wroclaw University of Economics (2009)

Pawlak, Z.: Granularity of knowledge, indiscernibility and rough sets. In: IEEE World Congress on Computational Intelligence, Fuzzy Systems Proceedings, vol. 1 (1998)

Russell, S., Norvig, P.: Artificial Intelligence. A Modern Approach. Pearson Education International (2003)

Turban, E., Leidner, D., Mclean, E., Wetherbe, J.: Information Technology for Management. In: Transforming Organizations in the Digital Economy, John Wiley \& Sons, Chichester (2008)

Yiming, Y., Tsostsos, J.: Knowledge granularity spectrum, action pyramid, and the scaling problem. International Journal of Pattern Recognition and Artifcial Intelligence 15(3) (2001)

Zadeh, L.A.: Toward a theory of fuzzy information granulation and its centrality in human reasoning and fuzzy logic. Fuzzy Sets and System 19(1) (1997)

Zhao, M., Yang, Q., Gao, D.: Axiomatic Definition of Knowledge Granularity and its Constructive Method. In: Wang, G., Li, T., Grzymala-Busse, J.W., Miao, D., Skowron, A., Yao, Y. (eds.) RSKT 2008. LNCS (LNAI), vol. 5009, pp. 348-354. Springer, Heidelberg (2008)

Zhuge, H.: The Knowledge Grid. World Scientific, Singapore (2004) 